\title{
Detection of continental-scale intensification of hourly rainfall extremes
}

\author{
Authors: Selma B Guerreiro* 1 , Hayley J Fowler ${ }^{1}$, Renaud Barbero ${ }^{1,2}$, Seth Westra ${ }^{3}$, Geert \\ Lenderink ${ }^{4}$, Stephen Blenkinsop ${ }^{1}$, Elizabeth Lewis ${ }^{1}$, Xiao-Feng Li $^{1}$ \\ 1 - School of Engineering, Newcastle University, UK \\ 2 - Irstea, Mediterranean Ecosystems and Risks, France \\ 3 -School of Civil, Environmental and Mining Engineering, University of Adelaide, Australia \\ 4 - Royal Netherlands Meteorological Institute, Netherlands \\ *Corresponding author: selma.guerreiro@ncl.ac.uk
}

\section{Introductory paragraph}

Temperature scaling studies suggest that hourly rainfall magnitudes might increase beyond thermodynamic expectations with global warming ${ }^{1,2,3}$; that is, above the Clausius-Clapeyron (CC) rate of $\sim 6.5 \%{ }^{\circ} \mathrm{C}^{-1}$. However, there is limited evidence of such increases in long-term observations. Here, we calculate continental-average changes in the magnitude and frequency of extreme hourly and daily rainfall observations from Australia over 1990-2013 and 19661989. Observed changes are compared to the uncertainty from natural variability and to expected changes from CC-scaling as a result of global mean surface temperature change. We show that increases in daily rainfall extremes are consistent with CC scaling, but are within the range of natural variability. By contrast, changes in the magnitude of hourly rainfall extremes are close to or exceed double the expected CC-scaling, and are above the range of natural variability, exceeding $3 \times \mathrm{CC}$ in the tropical region (north of $23^{\circ} \mathrm{S}$ ). These continental-scale changes in extreme rainfall are not explained by changes in the El Niño-Southern Oscillation or changes in the seasonality of extremes. Our results indicate that CC-scaling on temperature provides a severe underestimate of observed changes in hourly rainfall extremes in Australia, with implications for assessing the impacts of extreme rainfall.

\section{Main}

A warming climate is expected to cause an intensification of heavy rainfall ${ }^{4}$. Basic physical arguments suggest that, in the absence of changes in large-scale circulation (and associated moisture advection), the intensification will follow the water holding capacity of air, dictated 
by the laws of thermodynamics at a rate of $\sim 6.5 \%{ }^{\circ} \mathrm{C}^{-1}-$ termed the Clausius-Clapeyron (CC) rate $^{4}$. However, at regional scales, dynamical responses to climate change can cause deviation from the thermodynamic response ${ }^{5}$. Global and regional trend detection studies based on observations have reported on increases in daily rainfall extremes ${ }^{6}$, consistent with CC-scaling with global mean temperature ${ }^{7}$, despite the difficulties of detection and attribution to a warming climate ${ }^{8}$. However, evaluating the effects of warming on rainfall extremes is difficult in observed datasets due to limitations such as data inhomogeneities and missing data, lowdensity networks and inherent natural variability ${ }^{9,10}$. Since most hourly rainfall records are even shorter than their daily counterparts, establishing relationships between warming and increases in sub-daily magnitudes is particularly challenging ${ }^{10,11,12}$. Yet this is necessary for future planning since sub-daily rainfall observations are used extensively in engineering practice and infrastructure design; while daily extremes can induce river flooding (depending on antecedent conditions), hourly (and multi-hourly) extremes are often associated with urban flooding, flooding in small, steep catchments, and landslides ${ }^{19}$.

Attention has therefore focused on analysing and extrapolating observed extreme rainfalltemperature scaling relationships in the hope of obtaining more robust projections of extreme rainfall that exploit higher confidence in temperature projections ${ }^{13}$ and expected changes in the moisture budget. While increasing trends in sub-daily rainfall extremes have been observed for some regions ${ }^{9,10,14}$, observational extreme rainfall-temperature scaling studies on hourly timescales have reported on various rates of change, from negative in the tropics and sub-tropics ${ }^{15}$ to super-CC (more than CC) scaling rates ${ }^{2,3}$ in mid latitudes. The few climate model experiments at convection-permitting scales indicate a future intensification of hourly extreme rainfall in the mid-latitudes, with a CC or slightly above CC response ${ }^{16,17}$.

Given the lack of agreement between observed rainfall scaling rates based on day-to-day temperature variations and observed trends in rainfall extremes, it is clear that future predictions cannot be straightforwardly explained or derived from scaling relationships. There are several caveats to this method; temperature does not vary in proportion to specific humidity in many parts of the world ${ }^{13}$, which may cause observed rainfall scaling rates to show an inflection point above which they become negative - although using dew-point temperature to account for absolute humidity removes the inflection ${ }^{2}$. Changes in large-scale circulation patterns ${ }^{5}$ (due to climate change, anthropogenic aerosol forcing or other reasons) and local moisture availability through moisture convergence and upward motions ${ }^{18}{ }^{19}$ can have a significant impact on extreme rainfall ${ }^{15,18}$ and are not directly accounted for in scaling analyses. For e.g. strong cyclonic activity can cause lower temperatures as well as high rainfall 
amounts ${ }^{20}$, high-pressure systems generally cause relatively dry weather with high temperatures ${ }^{21}$ while local-scale dynamics can enhance scaling ${ }^{3,11}$. Changes in cloud size and the degree of mesoscale organization ${ }^{22}$ collectively affect extreme rainfall intensities and are not explicitly considered in the scaling approach. Using local temperature for scaling does not account for the possibility of remote moisture sources related to large-scale circulation ${ }^{12}$ or sea surface temperatures. To overcome this, some studies have used global or continental temperatures for scaling 7,10 , as a robust proxy of the moisture budget.

If we assume that extreme rainfall scales at the CC-rate and considering a global mean surface temperature (GMST) increase of $0.85^{\circ} \mathrm{C}$ for $1880-2012^{23}$, then methods of detection need to be sensitive to magnitude changes in the order of around $6 \%$ since pre-industrial times. Therefore, in both modelling and observational studies, spatial aggregation has often been used to identify a clearer signal in extreme rainfall from internal/natural variability (natural cycles and stochastic/random noise) $)^{7,24}$, with detection of trends in extreme rainfall only possible at global or continental scales ${ }^{8}$. Most hourly scaling studies have also used pooling of gauges to maximise the signal to noise ratio ${ }^{15,25}$ but, to date, over relatively small regions.

Considering the poor signal to noise ratio in point-based rainfall extreme time series, we therefore use the approach suggested by Fischer and Knutti ${ }^{24}$, which examines changes in the whole tail of the rainfall distribution rather than for individual quantiles, while using spatial aggregation at continental scales to identify a clearer signal in extreme rainfall from internal variability. Using this method, they were able to establish that frequency changes in daily extreme rainfall for observations and models follow theoretical CC scaling over the US and Europe in recent decades. We extend their work here to explore changes in hourly and daily extreme rainfall over the Australian continent between 1966-1989 and 1990-2013, and examine to what extent these are detectable from background internal variability. This study provides the first results of changes to hourly rainfall extremes aggregated to the continentalscale.

The Australian rainfall dataset has been extensively used in both trend analysis and temperature scaling studies. Westra and Sisson ${ }^{9}$ found increasing trends in hourly annual maxima from $1965-2005$ of $4.6 \%$ on average, but no statistically significant trends for daily data, with trends at different durations related to the seasonality of annual maxima ${ }^{26}$. Temperature scaling studies reveal negative scaling rates on local surface air temperature in tropical north Australia and positive (sometimes super-CC) rates in southern regions ${ }^{15,20}$. If dew point temperature is instead used as the scaling variable then the scaling changes to become slightly above $\mathrm{CC}\left(9 \%{ }^{\circ} \mathrm{C}^{-1}\right)$ in Darwin in tropical north Australia ${ }^{27}$. 
Here, we assess changes in the magnitude and frequency of extreme daily and hourly rainfall between 1966-1989 and 1990-2013. To do so, we ranked daily/hourly magnitudes at each gauge, which we call the "K-largest" values (i.e. K1 is the largest ranked value and K10 is the $10^{\text {th }}$ largest ranked value). To assess changes in magnitude, we binned all values (with 20 ranked values per bin) and calculated the continental mean changes in the mean of each bin. Then we calculated expected changes based on CC-scaling, using the change in GMST $\left(0.48^{\circ} \mathrm{C}\right)$. Using regional temperatures or using a different temperature dataset leads to smaller temperature changes and therefore higher dependencies of rainfall intensity, which strengthens our conclusions (see section 5 of Supplementary Information (SI)). The "data \& methods" section provides details on the dataset, the relationships between K-largest, all-day quantiles and absolute rainfall values and the methods for calculating magnitude changes. Changes in frequency - which show similar results to changes in magnitude are shown in SI (section 6).

We find that the magnitude of extreme daily rainfall is increasing in line with CC-scaling, with the most extreme rainfall showing larger changes (see Figure 1a). This is in line with results from Fischer and Knutti ${ }^{24}$ for daily rainfall observations over the US and Europe. Nevertheless, as internal variability is high, even when aggregated to the continental-scale, these changes are still within the range of the changes expected due to internal variability (grey shaded area in Figure 1a).

More striking is the large increase in the magnitude of hourly rainfall extremes at the continental-scale. Here, all changes are outside the range expected from internal variability and the increases are much higher than expected from CC-scaling (Figure 1b), with more extreme values showing the largest changes. The lower magnitudes (K140 to K100) show double the expected rate of increase from CC-scaling $(2 \times C C)$ while the highest magnitudes (K40 to $\mathrm{K} 20$ ) reach three times the expected rate of change (3xCC).

We note that using a smaller number of gauges to estimate the spatial mean can result in substantially different (even negative) spatial mean changes (see Section 3 of SI). The wide range of changes at the gauge-level (Figure 2) reflects the inherent large natural variability from short gauge (point) time series for extreme rainfall (which is superimposed on a forced response pattern).

To assess if there is a different pattern of behaviour between the tropical north (affected by tropical cyclones) and the mid-latitude climate of the south of Australia, we subdivide the gauges north or south of $23^{\circ} \mathrm{S}$ (Figure 3 ). We find that the behaviour for daily events is 
considerably different, with southern gauges showing changes well below expectations from CC-scaling; in contrast northern gauges are above $3 \times C C$. This explains the wide range of possible spatial changes when using sub-sets of the daily data shown in Figure S.2 of the SI. For hourly data, both north and south Australia show similar behaviour: changes are always above CC-scaling and increase for the larger magnitudes. Changes are lower in the southern gauges (around $2 \times C C$ ) and higher in wetter northern gauges (above $3 \times \mathrm{CC}$ for all K-bins).

El Niño Southern Oscillation (ENSO) is known to have an impact on Australian rainfall extremes ${ }^{9,28}$. Therefore, we next investigated the effect of different phases of ENSO on the occurrence of daily and hourly rainfall extremes using the Niño 3.4 index ${ }^{29}$. Differences in the distributions of each phase were tested using the Mann-Whitney-Wilcoxon test at the 0.05 significance level (see "Data \& Methods"). For daily rainfall, we found a higher probability of occurrence of extreme rainfall magnitudes during La Niña months than during other months (Figure 4). However, for hourly rainfall, both El Niño and La Niña months show a higher probability of occurrence of extreme magnitudes than months in the neutral phase. These results did not show any spatial pattern (Fig S.11 in SI). Since the number of months in El Niño and La Niña phases has decreased slightly in the second half of the analysis period (Table S.2), if ENSO was having a significant impact we would expect a decrease in the number of hourly extremes. Additionally, during the analysis period (1966-2013), we found no evidence for change points or trends within the Niño 3.4 index series or within its variance (see Section 8 of SI). Therefore, we argue that continental-scale changes in extreme rainfall presented here are not explained by changes in ENSO. We also tested the impacts of changes in the seasonality of rainfall extremes; this did not affect our results (see SI section 7). We did not consider impacts from aerosols (either direct or remote): possible uncertainties from aerosols are discussed in section 2 of the SI.

In this study, we have assessed changes in extreme hourly and daily rainfall magnitudes in Australia over the 1966-2013 period. We conclude that, at the daily scale, increases in magnitude over the whole of Australia are consistent with CC-scaling on the observed increase in GMST over the 1966-2013 period but are within the range of natural variability. At the hourly scale, however, increases are above 2 xCC scaling on GMST, outside the range of internal variability, and inconsistent with ENSO-forced variability. Since the spatial pattern of change is complex, aggregating to a continental-scale reduced the impact of locally relevant modes of variability and led to a higher signal-to-noise ratio. Therefore, for the first time, we were able to detect increases in hourly rainfall magnitudes and frequencies at the continental scale. While a dependency of hourly rainfall of up to 2 xCC based on short-term variability has 
been found in a limited number of studies, we here showed that this rate of intensification (and higher) can also be detected at the multi-decadal time scale when aggregating the response over a continent. Furthermore, the most extreme events show larger increases, consistent with latent heat release amplifying the most extreme events by the largest amount to reach super CC-scaling ${ }^{30}$.Therefore, we argue that there is a need to look at the whole tail of the distribution when assessing changes to extreme rainfall.

Our results show that, even at continental-scales, CC-scaling on temperature does not adequately explain observed climatological changes and therefore does not provide a reliable basis for projecting future changes to hourly rainfall extremes. Indeed, changes in atmospheric circulation patterns ${ }^{5}$, atmospheric stability, latent heat ${ }^{30}$, moisture convergence and upward motions ${ }^{18}{ }^{19}$, cloud size and the degree of mesoscale organization ${ }^{22}$, collectively affect extreme rainfall intensities and are not explicitly considered in the scaling approach. Further research into the different factors that influence extreme rainfall are clearly needed to project future changes with higher confidence. In the meantime, when pressed for projections of local future extreme rainfall, it is important to consider various sources of information besides CCscaling (e.g. climate models, observed dew point temperature scaling, observed trends or changes) at both local and regional scales. We recommend that a wide range of possible futures should be used to test existing and planned infrastructure, thus contributing to robust adaptation to climate change.

\section{Acknowledgements}

This work was supported by the INTENSE project. INTENSE is supported by the European Research Council (grant ERC-2013-CoG-617329). Hayley Fowler is funded by the Wolfson Foundation and the Royal Society as a Royal Society Wolfson Research Merit Award holder (grant WM140025). Seth Westra is supported by Australian Research Council Discovery project DP150100411.

\section{Author contributions}

SBG carried out the analysis. SBG, HJF and RB contributed to the design of the methodology. All authors discussed the results and contributed to writing the paper. 


\section{References}

1. .............. Lenderink G, Mok HY, Lee TC, Van Oldenborgh GJ. Scaling and trends of hourly precipitation extremes in two different climate zones - Hong Kong and the Netherlands. Hydrology and Earth System Sciences 2011, 15(9): 3033-3041.

2. ..... Lenderink G, Van Meijgaard E. Linking increases in hourly precipitation extremes to atmospheric temperature and moisture changes. Environmental Research Letters 2010, $5(2)$.

3.Berg P, Moseley C, Haerter JO. Strong increase in convective precipitation in response to higher temperatures. Nature Geosci 2013, 6(3): 181-185.

4. ..........Allan RP, Soden BJ. Atmospheric Warming and the Amplification of Precipitation Extremes. Science 2008, 321(5895): 1481-1484.

5. ...... Pfahl S, Ogorman PA, Fischer EM. Understanding the regional pattern of projected future changes in extreme precipitation. Nature Clim Change 2017, 7(6): 423-427.

6. Donat MG, Alexander LV, Yang H, Durre I, Vose R, Dunn RJH, et al. Updated analyses of temperature and precipitation extreme indices since the beginning of the twentieth century: The HadEX2 dataset. Journal of Geophysical Research: Atmospheres 2013, 118(5): 2098-2118.

7. Westra S, Alexander LV, Zwiers FW. Global Increasing Trends in Annual Maximum Daily Precipitation. Journal of Climate 2013, 26(11): 3904-3918.

8. ...............Min S-K, Zhang X, Zwiers FW, Hegerl GC. Human contribution to more-intense precipitation extremes. Nature 2011, 470(7334): 378-381.

9.Westra S, Sisson SA. Detection of non-stationarity in precipitation extremes using a maxstable process model. Journal of Hydrology 2011, 406(1): 119-128.

10. Barbero R, Fowler HJ, Lenderink G, Blenkinsop S. Is the intensification of precipitation extremes with global warming better detected at hourly than daily resolutions? Geophysical Research Letters 2017, 44(2): 974-983.

11. . Lenderink G, Fowler HJ. Hydroclimate: Understanding rainfall extremes. Nature Clim Change 2017, 7(6): 391-393.

12.Zhang X, Zwiers FW, Li G, Wan H, Cannon AJ. Complexity in estimating past and future extreme short-duration rainfall. Nature Geosci 2017, 10(4): 255-259.

13.Westra S, Fowler HJ, Evans JP, Alexander LV, Berg P, Johnson F, et al. Future changes to the intensity and frequency of short-duration extreme rainfall. Reviews of Geophysics 2014, 52(3): 522-555.

14.Utsumi N, Seto $\mathrm{S}$, Kanae $\mathrm{S}$, Maeda EE, Oki T. Does higher surface temperature intensify extreme precipitation? Geophysical Research Letters 2011, 38(16): n/a-n/a. 
15. Hardwick Jones R, Westra S, Sharma A. Observed relationships between extreme subdaily precipitation, surface temperature, and relative humidity. Geophysical Research Letters 2010, 37(22): n/a-n/a.

16. .Kendon EJ, Roberts NM, Fowler HJ, Roberts MJ, Chan SC, Senior CA. Heavier summer downpours with climate change revealed by weather forecast resolution model. Nature Climate Change 2014, 4(7): 570-576.

17 Prein AF, Rasmussen RM, Ikeda K, Liu C, Clark MP, Holland GJ. The future intensification of hourly precipitation extremes. Nature Clim Change 2017, 7(1): 48-52.

18. Loriaux JM, Lenderink G, Siebesma AP. Large-Scale Controls on Extreme Precipitation. Journal of Climate 2017, 30(3): 955-968.

19. .... Lenderink G, Barbero R, Loriaux JM, Fowler HJ. Super-Clausius-Clapeyron Scaling of Extreme Hourly Convective Precipitation and Its Relation to Large-Scale Atmospheric Conditions. Journal of Climate 2017, 30(15): 6037-6052.

20.Bao J, Sherwood SC, Alexander LV, Evans JP. Future increases in extreme precipitation exceed observed scaling rates. Nature Clim Change 2017, 7(2): 128-132.

21. .Chan SC, Kendon EJ, Roberts NM, Fowler HJ, Blenkinsop S. Downturn in scaling of UK extreme rainfall with temperature for future hottest days. Nature Geosci 2016, 9(1): 2428.

22. ... Lochbihler K, Lenderink G, Siebesma AP. The spatial extent of rainfall events and its relation to precipitation scaling. Geophysical Research Letters 2017, 44(16): 8629-8636.

23.IPCC. Climate Change 2013: The Physical Science Basis. Contribution of Working Group I to the Fifth Assessment Report of the Intergovernmental Panel on Climate Change.

[Stocker, T.F., D. Qin, G.-K. Plattner, M. Tignor, S.K. Allen, J. Boschung, A. Nauels, Y. Xia, V. Bex and P.M. Midgley (eds)] Cambridge University Press, Cambridge, United Kingdom and New York, NY, USA; 2013.

24.Fischer EM, Knutti R. Observed heavy precipitation increase confirms theory and early models. Nature Clim Change 2016, 6(11): 986-991.

25. Blenkinsop S, Chan SC, Kendon EJ, Roberts NM, Fowler HJ. Temperature influences on intense UK hourly precipitation and dependency on large-scale circulation. Environmental Research Letters 2015, 10(5): 054021.

26. .... Zheng F, Westra S, Leonard M. Opposing local precipitation extremes. Nature Clim Change 2015, 5(5): 389-390.

27. ...... Barbero R, Westra S, Lenderink G, Fowler HJ. Temperature-extreme precipitation scaling: a two-way causality? Int J Climatol 2018, 38: e1274-e1279.

28. Risbey JS, Pook MJ, McIntosh PC, Wheeler MC, Hendon HH. On the Remote Drivers of Rainfall Variability in Australia. Monthly Weather Review 2009, 137(10): 3233-3253. 
29. ...Rayner NA, Parker DE, Horton EB, Folland CK, Alexander LV, Rowell DP, et al. Global analyses of sea surface temperature, sea ice, and night marine air temperature since the late nineteenth century. Journal of Geophysical Research: Atmospheres 2003, 108(D14): n/a-n/a.

30.Pendergrass AG. What precipitation is extreme? Science 2018, 360(6393): 1072-1073. 


\section{Figure captions:}

Figure 1 - Changes in magnitude of daily and hourly rainfall for different definitions of extreme rainfall. Changes (differences) in magnitude (in $\mathrm{mm}$ ) of daily (a) and hourly (b) for different K-largest values. Changes are shown for: observations (blue line), expected changes based on the Clausius-Clapeyron (CC) scaling (red dashes), double the expected changes based on CC-scaling (CCX2 - red dotdashes) and expected changes from triple CC-scaling (CCX3 - red dots). The shaded grey area shows the changes expected due to internal variability using a $99^{\text {th }}$ confidence interval (calculated using a bootstrapping technique on the observed dataset, i.e. timeseries were randomly reshuffled, with replacement and retaining spatial correlation, and then divided into two sub-periods and changes calculated: the procedure was repeated 1000 times). All changes are shown as spatial means of 107 gauge records across Australia between 1990-2013 and 1966-1989. The labels in the figures show the lower end of the bins (i.e. K-largest 20 - K20 - shows the mean change of the 20 largest values, K40 shows the mean change of the values between $K 21$ and $K 40$ - including $K 40$, etc.)

Figure 2 - Changes in magnitude of extreme daily and hourly rainfall. Observed changes (differences) in the magnitude of the mean of the 20 largest values (K20) between 1990-2013 and 1966-1989 for a) daily and b) hourly rainfall.

Figure 3 - Changes in magnitude for northern and southern Australian daily and hourly rainfall for different definitions of extreme rainfall. Changes (differences) in magnitude (in $\mathrm{mm}$ ) of daily (a) and hourly (b) $\mathrm{K}$-largest values. Solid lines show changes in observed data, dashes show expected changes from CC-scaling (CC), dotdashes show expected changes from double CC-scaling (CCX2) and dot show expected changes from triple CC-scaling (CCX3). Results are shown for gauges north (orange) and south (purple) of $23^{\circ} \mathrm{S}$. All changes are shown as spatial means between 1990-2013 and 1966-1989. The x-axis labels show the lower end of the bins (i.e. $K$-largest $20-K 20$ - shows the mean change of the 20 largest values, $K 40$ shows the mean change of the values between $K 21$ and $K 40$ - including $K 40)$.

Figure 4-Effect of the phase of EI Niño-Southern Oscillation on the occurrence of extremes. Percentage of El Niño (red), La Niña (blue) and neutral (grey) months that have extreme daily (a) and hourly (b) rainfall, for different $K$-largest bins. The boxplots show the spread of the different gauges (boxes show the interquartile range with the median, whiskers extend to 1.5 times the interquartile range and values above or below this are shown as dots). The labels show the lower end of the bins (i.e. K20-shows the percentage of nino/nina/neutral months with values above or equal to K20, K40 shows the percentage of months with values between $K 40$ (inclusive) and $\mathrm{K} 20$ (exclusive)). 


\section{Data \& Methods}

Data availability

The data that support the findings of this study are available from the Australian Bureau of Meteorology upon request.

\section{Dataset}

The dataset used in this study was Australia's pluviograph record (temporal resolution of $5 \mathrm{~min}$ ) with 1493 gauges available. The Australian Bureau of Meteorology have quality controlled the data and data marked as "missing value recorded as zero rainfall" or "value estimated through linear interpolation as the rainfall recorded for that period was an accumulation" were dismissed. This dataset has been extensively used for trend analysis and CC-scaling studies $915,20,26,31,32$. Furthermore, Westra and Sisson ${ }^{9}$ performed an in depth investigation of the possible impacts of changes in the rainfall gauge equipment for the dataset used in this paper using three separate methods:

1) For each gauge, they assessed the impact of fitting a univariate non-stationary GEV model with both trend and step-change covariates with the date of the step change selected based on the recorded date of the gauge change

2) Fitted a max-stable process to the original series and to an adjusted series - where the change points identified in 1) had been removed

3) Applied a spatial GEV model to both the whole record and to a period before almost all equipment changes and compared the results

All the three separate methods showed that equipment changes had no noticeable impact on the non-stationarity of extreme rainfall.

To assess changes through time, we had to find a compromise between data length, number of gauges and amount of missing data. We therefore selected gauges with data between 1966 and 2013 with less than 30\% of missing data in each period of analysis (1966-1989 and 1990-2013). This resulted in 107 gauges available to the study.

Daily rainfall was obtained by summing hourly rainfall over each calendar day. Hourly data was declustered by selecting the maximum hourly rainfall in each calendar day. This means the comparison between daily and hourly changes is based on the same sample sizes and they are both constrained on calendar days, making it a fair comparison. 


\section{K-largest}

Due to the large variety of climatic regions in Australia, defining which quantiles are of interest presents difficulties since some gauges have limited or no rainfall even at high daily and hourly quantiles (like 0.95). Using wet-day/wet-hour quantiles is also not advisable since changes in the frequency of wet days/hours may interfere with changes in their magnitude ${ }^{33}$. Therefore, we used a K-largest approach (e.g.: $\mathrm{K} 1$ is the largest value, $\mathrm{K} 10$ the $10^{\text {th }}$ largest value) to assess changes in the magnitude and frequency of extreme daily and hourly rainfall.

Figure S.1 shows the range of rainfall magnitude associated with each K-largest, and their equivalence with quantiles.

\section{Magnitude changes}

Changes in magnitude were calculated according to the following steps, which are based on the methodology used in Fischer and Knutti ${ }^{24}$.

1. Rank values at each gauge for both periods. We refer to the ranked values as Klargest (i.e. $\mathrm{K} 1$ is the largest ranked value, $\mathrm{K} 20$ is the $20^{\text {th }}$ largest ranked value, at each gauge).

2. Bin values bounded by every $20 \mathrm{~K}$-largest and calculate the mean for each bin and each period:

$$
M_{k, p}=\operatorname{Mean}\left(M_{p}\right), M_{p} \in\left[M_{k, p}, M_{k-19, p}\right] \quad \text { (Eq.1) }
$$

3. Calculate absolute magnitude changes for each gauge and each bin:

With:

$M-$ magnitude

$\mathrm{k}$ - K-largest

$\mathrm{p}$ - period

2 - second period (1990-2013)

1 - first period (1966-1989)

$$
M_{k, 2}-M_{k, 1} \quad(\text { Eq.2) }
$$

4. Calculate the spatial mean (all gauges) for each K-largest bin.

5. To calculate expected changes from CC-scaling: multiply period 1 time-series by a change factor (3.1\%) based on the Global Mean Temperature change (GISS Surface Temperature Analysis ${ }^{34,35}$ - see Section 5 of Supplementary Information) between the two periods and the $\mathrm{CC}$-rate $\left(6.5 \% /{ }^{\circ} \mathrm{C}\right)$, and repeat steps 2 to 4 .

6. For $2 \times C C$-scaling: use the same method as step 5 but using a change factor of $6.2 \%$. 


\section{Frequency changes}

Changes in frequency were calculated according to the following steps, which are based on the methodology used in Fischer and Knutti ${ }^{24}$.

1. Calculate the magnitude of the K-largest $140,120,100,80,60,40,20$ for every gauge for period 1.

2. Calculate frequency changes for each gauge:

- Count number of values equal to or above each K-largest (defined in previous step for period 1$)$ for each period $\left(F_{k, p}\right)$

- Compute a correction factor based on length of missing data $\left(\mathrm{cf}_{\mathrm{k}, \mathrm{p}}\right)$ :

$$
c f_{k, p}=1+\frac{\text { number of missing values }}{\text { number of non missing values }}
$$

- Apply the correction factor and calculate the difference in number of values for each K-largest:

$$
F_{k, 2} \times c f_{k, 2}-F_{k, 1} \times c f_{k, 1} \quad \text { (Eq.4) }
$$

4. Calculate the spatial mean (all gauges) for each K-largest

5. To calculate expected changes from CC-scaling: multiply period 1 time-series by a change factor (3.1\%) based on the Global Mean Temperature change (GISS Surface Temperature Analysis ${ }^{34,35}$ - see Section 5 of Supplementary Information) between the two periods and the CC-rate $\left(6.5 \% /{ }^{\circ} \mathrm{C}\right)$, and repeat steps 2 to 4 .

6. For $2 \times C C$-scaling: use the same method as step 5 but using a change factor of $6.2 \%$.

\section{Internal variability analysis}

In order to test whether the magnitude and frequency changes exceed those expected from internal variability only, we applied a bootstrap technique using a Monte Carlo algorithm. We randomly resampled (with replacement) the gauge data (daily values) across the whole observational period (1966-2013) and divided each resampled time-series in two sub-periods. The analysis was then applied on the resampled sub-periods. The process was repeated 1000 times to derive a two-sided confidence interval, as a rough estimate for the changes expected due to internal variability. Within this bootstrap resampling, we retained the spatial correlation between sites by sampling on the same days across all gauges. The $99^{\text {th }}$ confidence interval of change refers to the quantiles of the changes of the resampled series correspondent to 0.01 and 0.99 probability. 


\section{ENSO}

The El Niño and the Southern Oscillation (ENSO) time-series ${ }^{29}$ was downloaded from https://www.esrl.noaa.gov/psd/gcos wgsp/Timeseries/Nino34/for the commonly used region Niño $3.4\left(5^{\circ} \mathrm{N}-5^{\circ} \mathrm{S}, 120^{\circ} \mathrm{W}-170^{\circ} \mathrm{W}\right)$. Following the NOAA ${ }^{36}$ definition, El Niño (La Niña) events were defined as 5 consecutive months where the 3-month rolling mean of ENSO anomalies (calculated for $1951-2000)$ are above (below) or equal to $+0.5(-0.5){ }^{\circ} \mathrm{C}$.

For each phase (EI Niño, La Niña and neutral) the number of months with rainfall values in each K-largest bin (see "magnitude changes" steps 1 and 2 above) were counted and divided by the total number of months in the respective phase. Mann-Whitney-Wilcoxon test at the 0.05 significance level was used to assess differences in the distributions of each ENSO phase. For daily rainfall, La Niña months are statistically distinct from neutral and El Niño months, while neutral and El Niño months are identical. For hourly rainfall, El Niño and La Niña show statistically identical distributions for $\mathrm{K} 140$ and $\mathrm{K} 20$ and are distinct for all other bins, while the neutral phase is always statistically distinct from the other two (Figure 4).

\section{Methods only references:}

31. .............. Wasko C, Sharma A. Steeper temporal distribution of rain intensity at higher temperatures within Australian storms. Nature Geosci 2015, 8(7): 527-529.

32. Wasko C, Sharma A, Westra S. Reduced spatial extent of extreme storms at higher temperatures. Geophysical Research Letters 2016, 43(8): 4026-4032.

33.Schär C, Ban N, Fischer EM, Rajczak J, Schmidli J, Frei C, et al. Percentile indices for assessing changes in heavy precipitation events. Climatic Change 2016, 137(1): 201-216.

34. ............ GISTEMP Team. ISS Surface Temperature Analysis (GISTEMP). NASA Goddard Institute for Space Studies. 2017 [cited 10-10-2017]Available from: https://data.giss.nasa.gov/gistemp/.

35. ..... Hanson S, Nicholls R, Ranger N, Hallegatte S, Corfee-Morlot J, Herweijer C, et al. A global ranking of port cities with high exposure to climate extremes. Climatic Change 2011, 104(1): 89-111.

36.NOAA. Equatorial Pacific Sea Surface Temperatures. 2017 [cited]Available from: https://www.ncdc.noaa.gov/teleconnections/enso/indicators/sst.php 


\section{Changes in magnitude (1990-2013 from 1966-1989)}

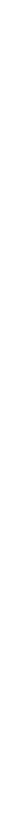

- observed - - CC $\cdot-\cdot \mathrm{CC} 2 \cdots \cdot \mathrm{CC} 33$ 
Magnitude changes for K20 (1990-2013 from 1966-1989)

a) Daily

b) Hourly

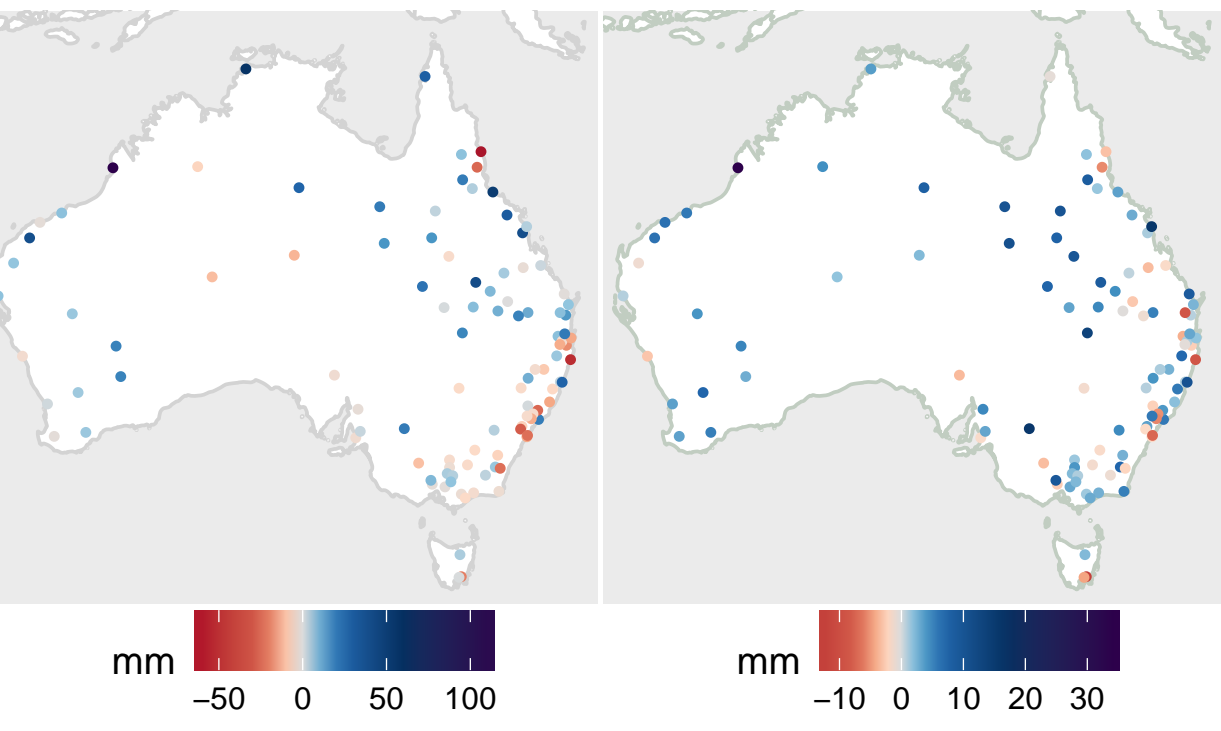


Effect of ENSO's phase on the occurrence of extremes
K140
K120
K100
$\mathrm{K} 80$
K60
K40
K20

อ $15-$

$\stackrel{\infty}{\frac{N}{C}} 10-\bullet$

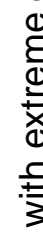
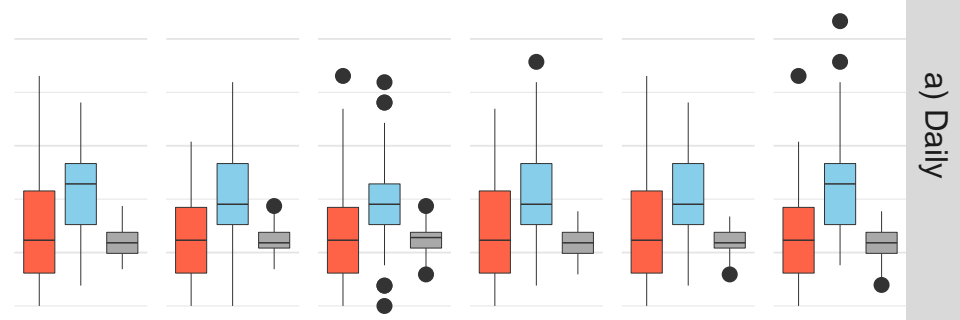

हृ

15 -

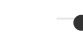

0
0
0
0
0
0
0
0
0
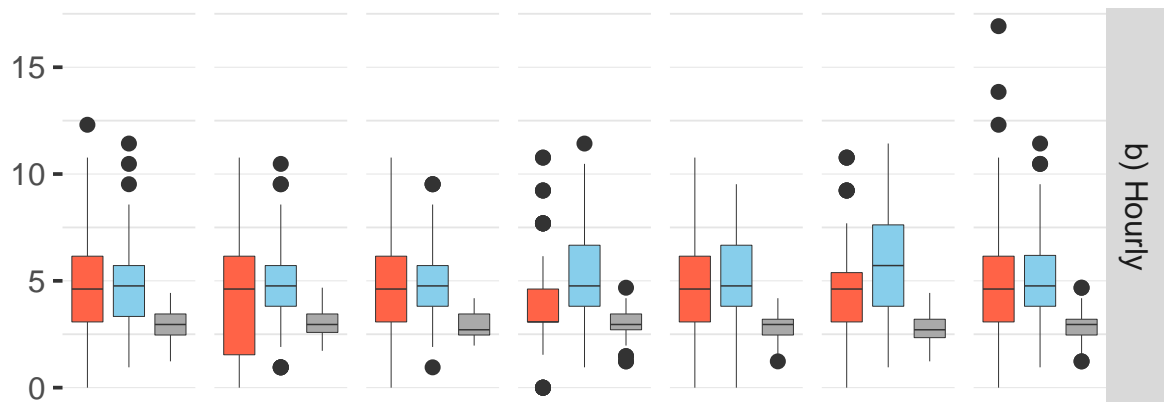

ENSO $\biguplus^{\prime}$ nino $\biguplus^{\prime}$ nina $\biguplus^{\prime}$ neutral 